\title{
The first practical approach to new pyrimido[4,5- $d]$ pyridazines: novel chemoselective and regioselective reactions
}

\author{
Bahador Karami,* Sedigheh Akrami, Saeed Khodabakhshi, and S. Setareh Rahmatzadeh \\ Department of Chemistry, Yasouj University, Yasouj, Zip Code: 75918-74831 P.O.Box 353, Iran \\ E-mail: karami@mail.yu.ac.ir
}

\begin{abstract}
A new three-component condensation of aryl glyoxals, acetylacetone and urea in the presence of a small amounts of tungstate sulfuric acid (TSA) leads to novel functionalized 5-acetyl-4-(aryloyl)3,4-dihydropyrimidinones which these heterocycles can undergo the Knorr condensation with hydrazines to produce new pyrimido[4,5- $d]$ pyridazines in good yields. These approaches are consistent with principles of green chemistry due to some factors, such as the use of a safe and recyclable catalyst under solvent-free conditions.
\end{abstract}

Keywords: Hydrazine, aryl glyoxal, dihydropyrimidinone, pyrimido[4,5-d]pyridazine.

\section{Introduction}

The strategies of multicomponent reactions (MCRs), especially three-component reactions, offer significant advantages over conventional linear-type syntheses because the combination of three components to generate new products in a single step is easy and extremely economic. ${ }^{1,2}$ MCRs are particularly useful to generate diverse chemical libraries of drug-like molecules for biological screening. ${ }^{3}$ A broad spectrum of biological activity have been reported for dihydropyrimidinones and pyridazine derivatives. ${ }^{4}$ For example, the pyridazine structure found within the structure of several pharmaceutical drugs such as cadralazine, ${ }^{5}$ minaprine, ${ }^{6}$ cefozopran, ${ }^{7}$ pipofezine (azaphen), ${ }^{8}$ and hydralazine. ${ }^{9}$ Due to the use of functionalized dihydropyrimidine-2-ones as potent calcium channel blockers, ${ }^{10}$ antihypertensive agents ${ }^{11}$ and neuropeptide $\mathrm{Y}$ antagonist ${ }^{12}$ and also their diversified activities such as antibacterial, ${ }^{13}$ antiviral, ${ }^{14}$ and antitumour properties, ${ }^{15}$ these heterocyclic compounds also play a significant role in synthetic, therapeutic and bioorganic chemistry.

\section{Results and Discussion}

According to the above-mentioned background and our programmatic interest on the heterocyclic synthesis, ${ }^{16-18}$ here, we developed a strategy to provide some novel 5-acetyl-4-(aryloyl)-3,4- 
dihydropyrimidinones 4 via a one-pot, and three-component condensation of acetylacetone (1), urea (2) and aryl glyoxals 3 (which in turn are obtained by selenium dioxide oxidation of the corresponding phenyl ketones) using tungstate sulfuric acid (TSA) as an efficient catalyst under solvent-free conditions (Scheme 1). It is interesting to note that these reactions are chemoselective and only one structural isomer would be formed. However, no trace was found for compounds $\mathbf{5}$ during the reaction processes by thin layer chromatography.

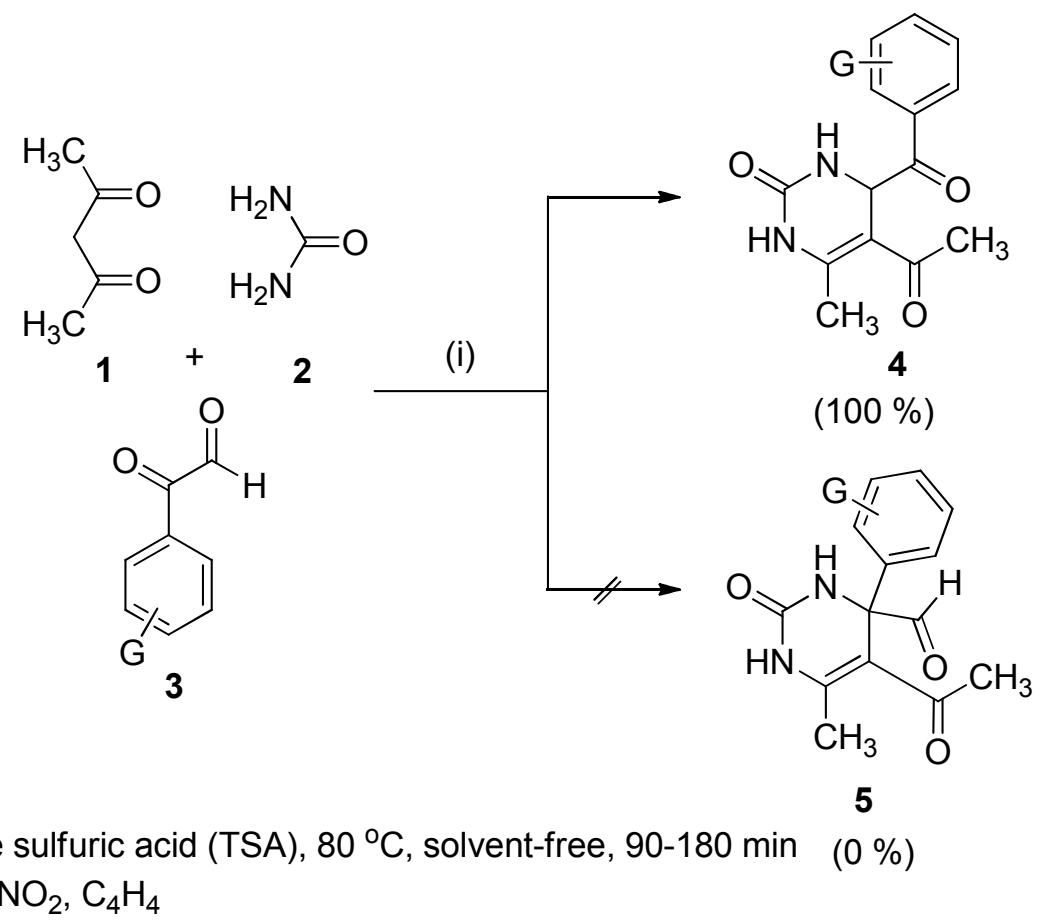

Scheme 1. Synthesis of 5-acetyl-4-(aryloyl)-3,4-dihydropyrimidinones.

The structures and purity of the obtained products were deduced from their infrared (IR), elemental analysis (CHN), and nuclear magnetic resonance (NMR) spectral data. As a suitable evidence for chemoselectivity of the reactions, the ${ }^{1} \mathrm{H}$ NMR (DMSO- $d_{6}, 400 \mathrm{MHz}$ ) spectrum of compound 4a exhibited two singlets of the nitrogen protons $(\delta=10.61$ and 10.10), a doublet $(\delta=7.35)$ and singlet signals $(\delta=7.20)$ corresponding to the aromatic protons. Also, due to the similar chemical shift, the resonance of the two methyl groups protons in the ${ }^{1} \mathrm{H}$ NMR spectrum of $\mathbf{4 a}$ appeared at $\delta=1.94$ as a singlet signal. A singlet signal at $\delta=5.55$ is the main reason for the formation of compounds 4 as the only isomer (Figure 1). After synthesis of novel 1,4-dicarbonyls 4 (Table 1), we found that these compounds participate in Knorr condensation with hydrazine derivatives 6 to produce new fused heterocycles such as pyrimido[4,5-d]pyridazines 7 (Scheme 2). We thought that two isomers may be formed. In regard of thin layer chromatography (TLC) experiments during the reaction progresses, we found that only one regioisomer has been formed. The regioselectivity of these reactions was also proved by NMR spectroscopy. For example, the ${ }^{1} \mathrm{H}$ NMR (DMSO- $d_{6}, 400 \mathrm{MHz}$ ) spectrum of $\mathbf{7 b}$ exhibited two sharp singlets identified as two methyl $(\delta=2.05)$ and $(\delta=2.08)$ 
protons. The multiplets $(\delta=7.15-7.55)$ corresponded to the protons of the two phenyl groups. The protons of NH groups also appeared as two distinct signals at $\delta=10.23$ and $\delta=10.64$ (Figure 2). The proton decoupled ${ }^{13} \mathrm{C}$ NMR spectrum of $7 \mathbf{b}$ showed 16 distinct resonances in agreement with the proposed structure. As can be seen in Figure 2, the disappearance of the signal for methine hydrogen can prove that the compounds $\mathbf{8}$ have not been formed.

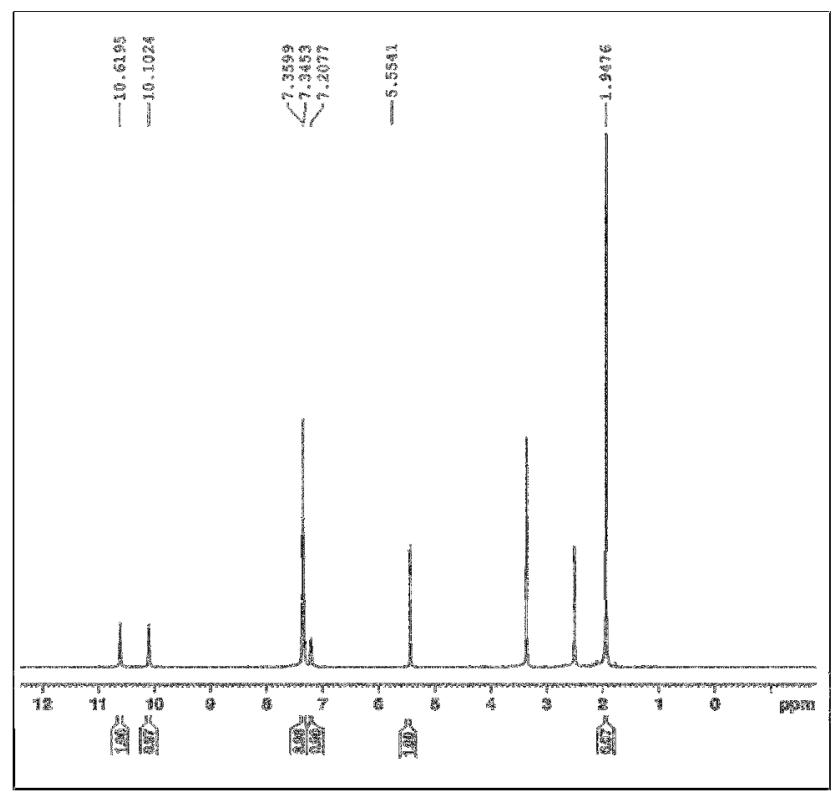

Figure 1. ${ }^{1} \mathrm{H}$ NMR spectrum of compound $4 \mathbf{a}$.

This type of procedure would allow chemists to prepare a large number of novel fused heterocycles which may be biologically active (Table 2). Minimizing catalyst loss and avoidance of organic solvents during chemical reactions requires a fundamental understanding of green chemistry factors. These concepts provide direction for improvements in organic synthesis and finishing of environmental and economic concerns. Organic synthesis based on green strategies has been widely investigated due to stringent environment and economic regulations. Not only the ecological profile (through helping to decrease hazardous industrial waste), but also the economic profile (through the elimination of expensive organic solvent) is further improved if the catalyst be recyclable and reaction conditions be solvent-free. ${ }^{19-21}$ It should be noted that our efforts for the present reactions without using catalyst were unsuccessful. In general, TSA has valuable and special features including: a) reaction carried out under solvent free conditions; b) high thermal stability; c) a small amount of recyclable catalyst used making this reaction economic and eco-friendly. In this process, the catalyst was recycled and reused for three cycles during which a little appreciable loss was observed in the catalytic activities. These unique features are main reason for using the TSA as catalyst. 


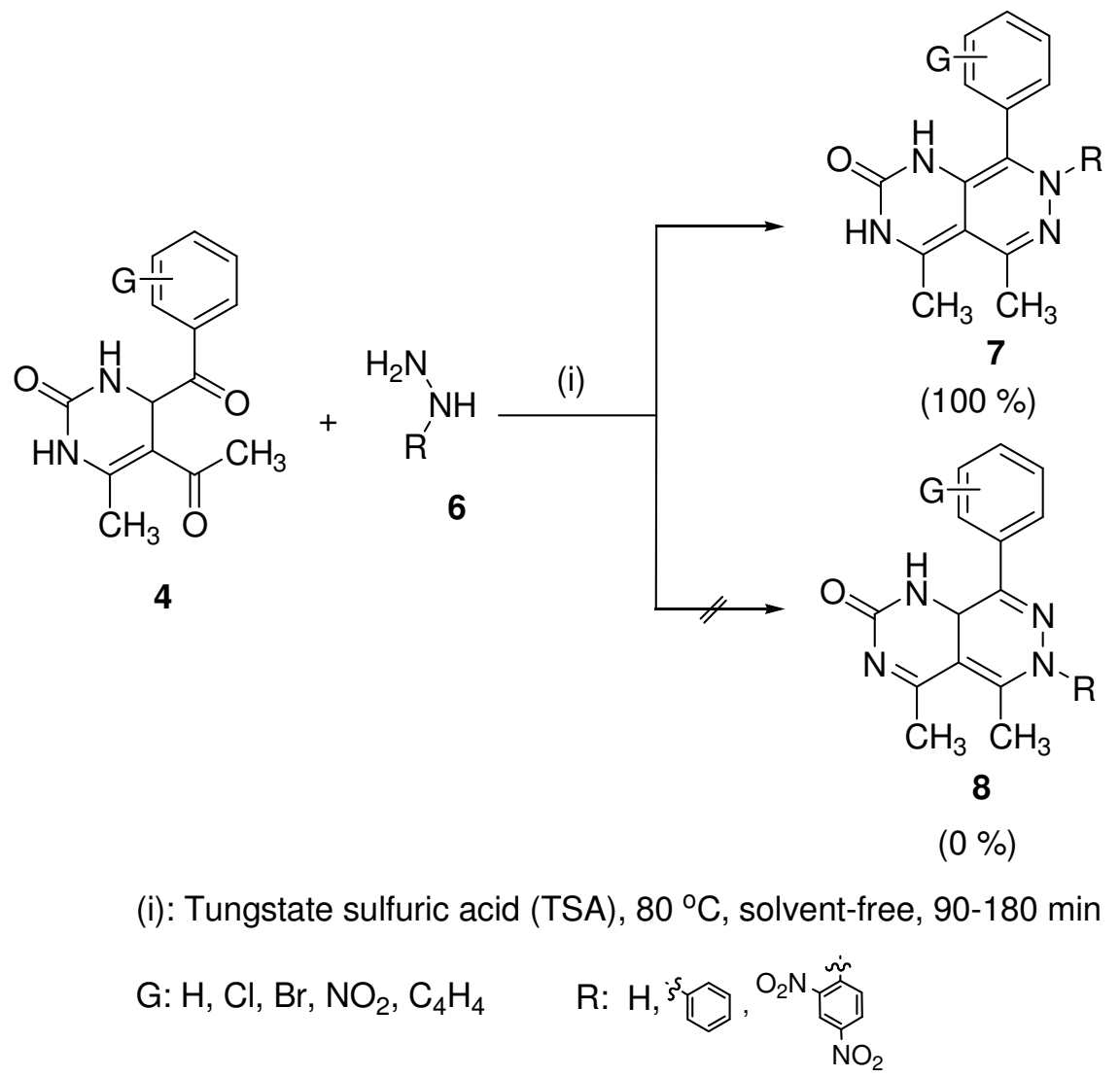

Scheme 2. Synthesis of pyrimido[4,5-d]pyridazines.

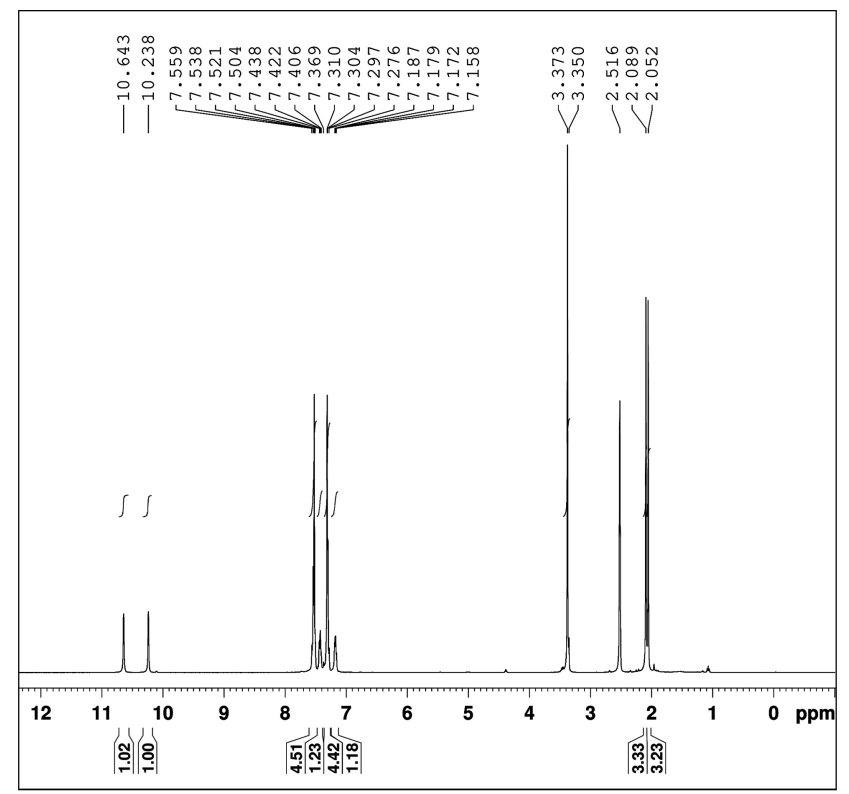

Figure 2. ${ }^{1} \mathrm{H}$ NMR spectrum of compound $\mathbf{7 b}$. 
Table 1. Synthesis of 5-acetyl-4-(aryloyl)-3,4-dihydropyrimidinones using TSA under solvent-free conditions

Entry

${ }^{\mathrm{a}}$ Refers to isolated yields.

The suggested mechanism for this two-step process has been shown in Scheme 3. Protonation of the hydroxyl group of arylglyoxal by a Brønsted acid (TSA) generates an electrophilic centre, which is easily attacked by the urea to form iminium intermediate $\mathbf{9}$ which is the key rate-limiting step.

Table 2. Synthesis of pyrimido[4,5- $d]$ pyridazines using TSA under solvent-free conditions

\begin{tabular}{llcc}
\hline Entry & Product & Yield $^{\mathrm{a}}(\%)$ & $\mathrm{mp}\left({ }^{\circ} \mathrm{C}\right)$ \\
\hline $7 a$ & & & \\
& & & \\
\end{tabular}


Table 2. Continued

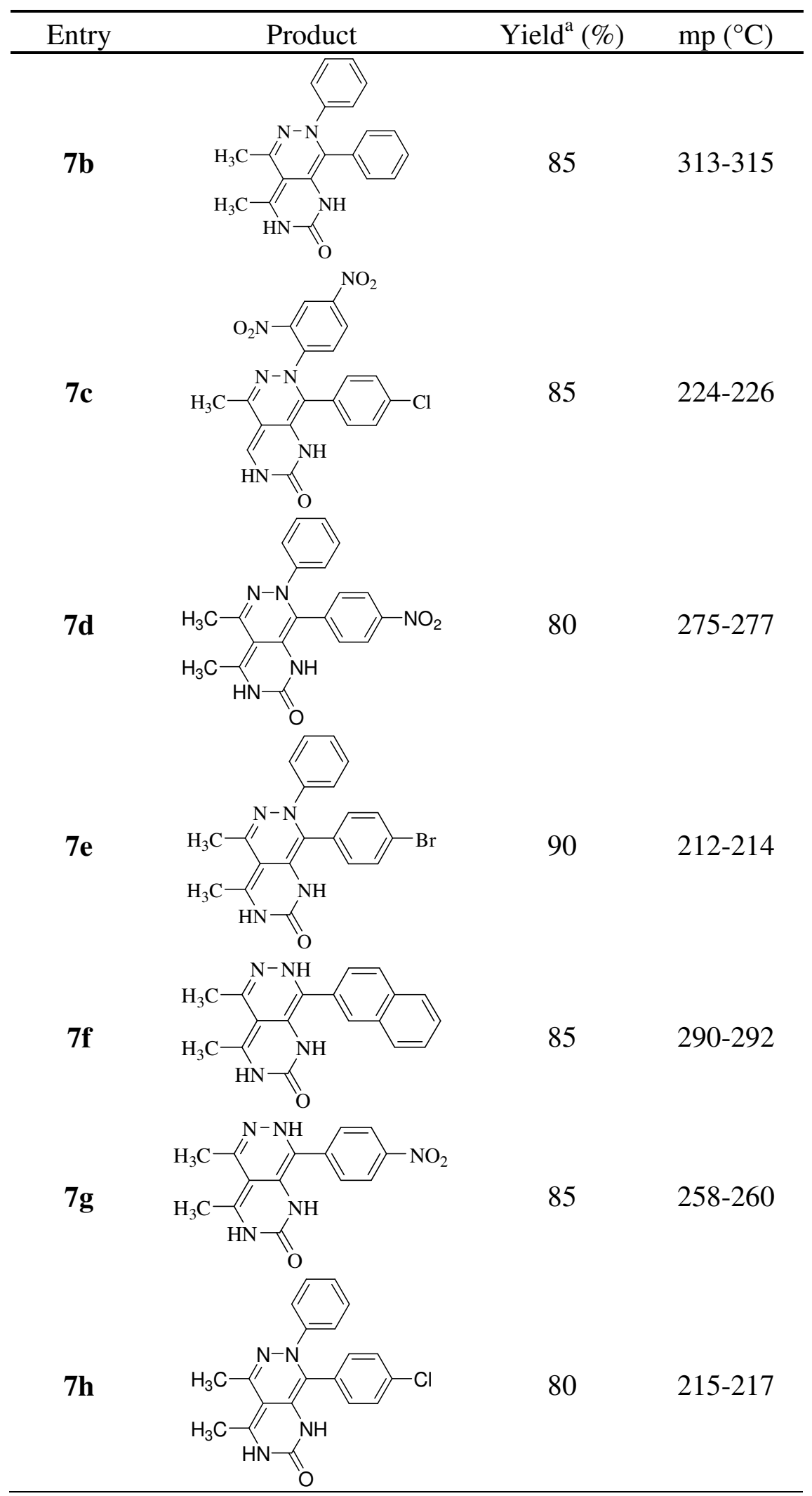

${ }^{a}$ Refers to isolated yields. 
Interception of this iminium intermediate $\mathbf{7}$ by $\beta$-dicarbonyl produces an open chain ureide 10, which subsequently cyclizes through dehydration process yielding compounds 4 . In the second step for the formation of pyrimido[4,5- $d$ ]pyridazines 7, the TSA also acts as an efficient acid so that it can release a proton and activates the $\delta$-dicaronyl, thus, the energy of the transition state decreases and the rate of the nucleophilic displacement increases. After nuclepphilic attack of the hydrazine to the activated carbonyls and dehydration, the final products 7 would be formed.

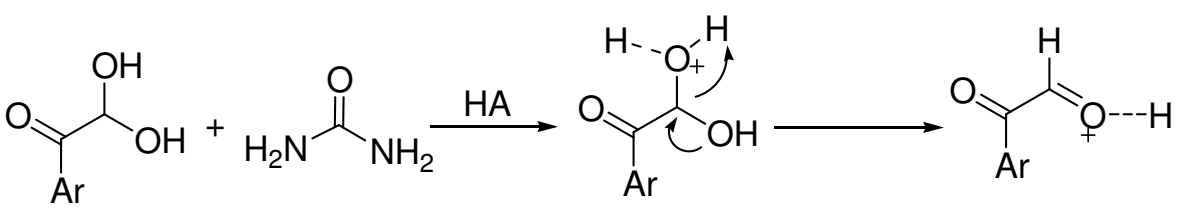

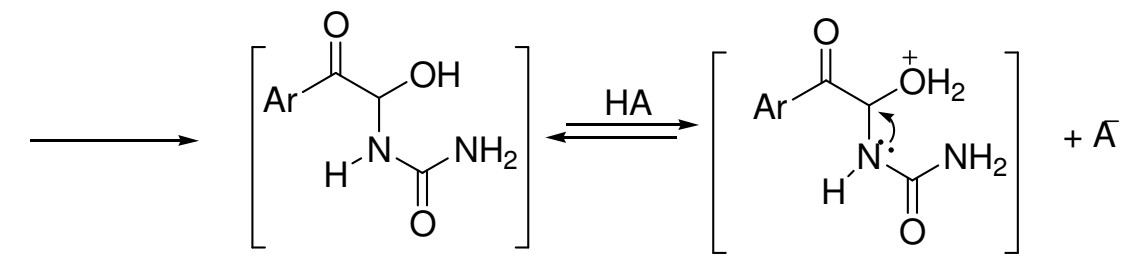<smiles>CC(=O)C=C(C)C(C)=O</smiles><smiles>CC(=O)C1=C(C)NC(=O)NC1C(C)=O</smiles>

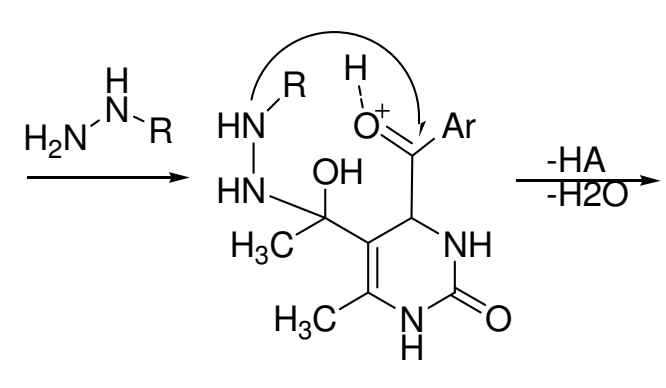

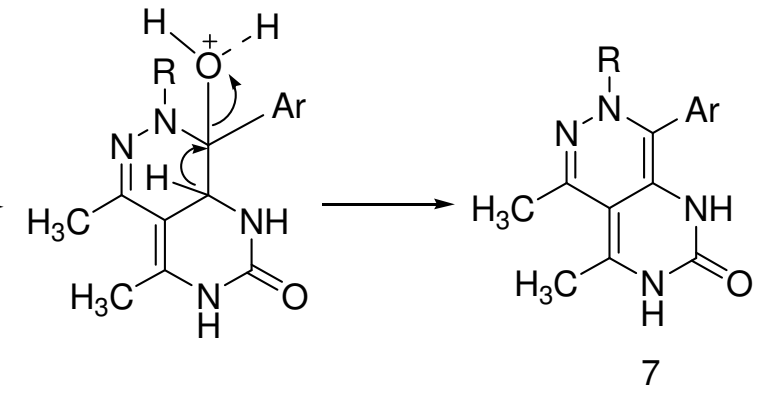<smiles>CCCOP(=O)(O)O[V](O)(O)(O)OS(=O)(=O)O</smiles>

Scheme 3. Plausible mechanism for the formation of compounds 4 and 7. 


\section{Conclusions}

The reaction of aryl glyoxals, urea, and acetylacetone in the presence of tungstate sulfuric acid provides a simple one-pot entry to novel functionalized 5-acetyl-4-(aryloyl)-3,4dihydropyrimidinones of potential synthetic and pharmaceutical interest. Besides, 5-acetyl-4(aryloyl)-3,4-dihydropyrimidinone derivatives can undergo the Knorr condensation with hydrazines to produce novel pyrimido[4,5- $d$ ]pyridazines. The use of a green and recyclable catalyst (TSA) under solvent-free conditions, high yield of pure products, short reaction times, and a simple workup procedure make the present method a valid contribution in accord with green chemistry principles. It is worthwhile to note that the presence of transformable functionalities in the products makes them potentially valuable for further synthetic manipulations.

\section{Experimental Section}

General. Aryl gloxals were synthesized in accord with report by Riley and Gray. ${ }^{22}$ Tungstate sulfuric acid was prepared in accord with our previous literature. ${ }^{23}$ Other chemicals were purchased from Merck and Aldrich. The reactions were monitored by TLC (silica gel $60 \mathrm{~F}_{254}$, hexane/EtOAc). IR spectra were recorded on a FT-IR JASCO-680 and the NMR spectra were obtained on a BrukerInstrument DPX-400 MHz Avance II model. The varioEl CHNS Isfahan Industrial University was used for elemental analysis.

5-Acetyl-4-(aryloyl)-3,4-dihydropyrimidinones 4. A mixture of acetyl acetone (1) (1 mmol), urea (2) $(1.5 \mathrm{mmol})$, aryl glyoxal $\mathbf{3}(1 \mathrm{mmol})$, and TSA $(0.05 \mathrm{mmol})$ was stirred and heated at $80{ }^{\circ} \mathrm{C}$ in a preheated oil bath for an appropriate time. After completion of the reaction as indicated by TLC (120-180 min), the reaction mixture was cooled to room temperature, then, washed with cold water and recrystallized from EtOH to afford the pure product 4 . The separated catalyst was washed with diethyl ether, dried at $70{ }^{\circ} \mathrm{C}$ for $45 \mathrm{~min}$, and reused in another reaction.

5-Acetyl-4-(benzoyl)-6-methyl-3,4-dihydropyrimidinone (4a). light yellow solid, IR (KBr): 3057, 1720, 1648, 1591, 1448, $1027 \mathrm{~cm}^{-1}$; ${ }^{1} \mathrm{H}$ NMR (DMSO- $d_{6}, 400 \mathrm{MHz}$ ): $\delta=10.61$ (s, $1 \mathrm{H}$ ), 10.10 (s, $1 \mathrm{H}), 7.35(\mathrm{~d}, 3 \mathrm{H}, J=5.6 \mathrm{~Hz}), 7.20(\mathrm{~s}, 1 \mathrm{H}), 5.55(\mathrm{~s}, 1 \mathrm{H}), 1.94(\mathrm{~s}, 6 \mathrm{H}) ;{ }^{13} \mathrm{C}$ NMR (DMSO- $d_{6}, 100$ MHz): $\delta=193.15,183.00,153.88,129.96,128.79,126.50,124.11,119.60,112.66,103.85,57.38$, 23.25; Anal. Calcd. for $\mathrm{C}_{14} \mathrm{H}_{14} \mathrm{~N}_{2} \mathrm{O}_{3}$ : C, 65.11; H, 5.46; N, 10.85. Found: C, 65.31; H, 5.40; N, 10.73 .

5-Acetyl-4-(2-naphthoyl)-6-methyl-3,4-dihydropyrimidinone (4b). Pale goldenrod solid, IR (KBr): 3289, 1721, 1693, 1606, 1422, $1210 \mathrm{~cm}^{-1} ;{ }^{1} \mathrm{H}$ NMR (DMSO- $\left.d_{6}, 400 \mathrm{MHz}\right): \delta=10.75(\mathrm{~s}$, $1 \mathrm{H}), 10.17(\mathrm{~s}, 1 \mathrm{H}), 7.91(\mathrm{~s}, 1 \mathrm{H}), 7.87-7.78(\mathrm{~m}, 3 \mathrm{H}), 7.53-7.43(\mathrm{~m}, 3 \mathrm{H}), 5.55(\mathrm{~s}, 1 \mathrm{H}), 1.97(\mathrm{~s}, 6 \mathrm{H})$;

${ }^{13} \mathrm{C}$ NMR (DMSO- $d_{6}, 100 \mathrm{MHz}$ ): $\delta=193.23,183.00,153.92,133.00,131.46,128.29,127.65$, 127.51, 126.64, 125.89, 122.51, 122.46, 119.76, 113.47, 103.90, 57.00, 23.33; Anal. Calcd. for $\mathrm{C}_{18} \mathrm{H}_{16} \mathrm{~N}_{2} \mathrm{O}_{3}: \mathrm{C}, 70.12 ; \mathrm{H}, 5.23 ; \mathrm{N}, 9.09$. Found: C, 70.28; H, 5.16; N, 8.98 . 
Pyrimido[4,5-d]pyridazines 7. A mixture of 4 (1 mmol), 6 (1 mmol) and TSA $(0.05 \mathrm{mmol})$ was stirred and heated at $80{ }^{\circ} \mathrm{C}$ in a preheated oil bath for an appropriate time (120-180 min). After completion of the reaction as indicated by TLC (EtOAc/ hexan, 1:4), the reaction mixture was dissolved in hot EtOH and catalyst was separated by filtration. The solvent was removed under vacuum and the products 7 were purified by recrystallization in EtOH.

4,5-Dimethyl-8-phenylpyrimido[4,5-d]pyridazin-2(1H,3H,7H)-one (7a). Pale yellow solid, IR (KBr): 3455, 3075, 2995, 2990, 2890, 1690, 1650, 1490, $1100 \mathrm{~cm}^{-1}$; ${ }^{1} \mathrm{H}$ NMR (DMSO- $d_{6}, 400$ MHz): $\delta=12.41(\mathrm{~s}, 1 \mathrm{H}), 10.64(\mathrm{~s}, 1 \mathrm{H}), 10.19(\mathrm{~s}, 1 \mathrm{H}), 7.14(\mathrm{dd}, 1 \mathrm{H}, J=8.4,4.4 \mathrm{~Hz}), 7.24(\mathrm{~d}, 4 \mathrm{H}, J$ $=4 \mathrm{~Hz}), 1.97(\mathrm{~s}, 1 \mathrm{H}) ;{ }^{13} \mathrm{C}$ NMR $\left(\mathrm{DMSO}-d_{6}, 100 \mathrm{MHz}\right): \delta=154.88,147.17,138.58,131.11,128.90$, 126.43, 124.62, 118.57, 111.71, 107.34, 12.80, 10.19; Anal. Calcd. for $\mathrm{C}_{13} \mathrm{H}_{12} \mathrm{~N}_{4} \mathrm{O}: \mathrm{C}, 64.99 ; \mathrm{H}$, 5.03; N, 23.32.. Found: C, 65.25; H, 4.89; N, 23.56.

4,5-Dimethyl-7,8-diphenylpyrimido[4,5- $d]$ pyridazin-2(1H,3H,7H)-one (7b). Light yellow solid, IR (KBr): 3450, 3030, 2995, 2990, 2890, 1690, 1650, 1490, $1100 \mathrm{~cm}^{-1}$; ${ }^{1} \mathrm{H}$ NMR (DMSO-d, 400 $\mathrm{MHz}): \delta=10.64(\mathrm{~s}, 1 \mathrm{H}), 10.23(\mathrm{~s}, 1 \mathrm{H}), 7.55-7.50(\mathrm{~m}, 4 \mathrm{H}), 7.43-7.36(\mathrm{~m}, 1 \mathrm{H}), 7.31-7.27(\mathrm{~m}, 4 \mathrm{H})$, 7.18-7.15 (m, 1H), $2.08(\mathrm{~s}, 3 \mathrm{H}), 2.05(\mathrm{~s}, 3 \mathrm{H}) ;{ }^{13} \mathrm{C}$ NMR (DMSO- $\left.d_{6}, 100 \mathrm{MHz}\right): \delta=154.72,148.09$, $139.75,138.80,130.90,129.72,129.05,129.05,127.85,126.69,124.81,124.56,119.31,110.78$, 110.76, 12.72, 11.97; Anal. Calcd. for $\mathrm{C}_{19} \mathrm{H}_{16} \mathrm{~N}_{4} \mathrm{O}$ : C, 72.13; H, 5.10; N, 17.71. Found: C, 71.98; H, $5.23 ; \mathrm{N}, 17.90$.

8-(4-Chlorophenyl)-7-(2,4-dinitrophenyl)-5-methylpyrimido[4,5- $d]$ pyridazin-2(1H,3H,7H)-one (7c). Light yellow, IR (KBr): 3420, 1689, 1545, 1504, 1382, $1345 \mathrm{~cm}^{-1} .{ }^{1} \mathrm{H}$ NMR (DMSO- $d_{6}, 400$ $\mathrm{MHz}): \delta=10.66(\mathrm{~s}, 1 \mathrm{H}), 10.17(\mathrm{~s}, 1 \mathrm{H}), 7.91-7.84(\mathrm{~m} 3 \mathrm{H}), 7.41-7.35(\mathrm{~m}, 4 \mathrm{H}), 1.94(\mathrm{~s}, 6 \mathrm{H}) \mathrm{cm}^{-1} ;{ }^{13} \mathrm{C}$ NMR (DMSO- $\left.d_{6}, 100 \mathrm{MHz}\right): \delta=154.04,147.44,144.50,136.78,136.32,136.10,133.00,132.61$, 129.21, 127.57, 124.29, 124.18, 115.20, 109.51, 12.21, $11.43 \mathrm{~cm}^{-1}$; Anal. Calcd. for $\mathrm{C}_{19} \mathrm{H}_{13} \mathrm{ClN}_{6} \mathrm{O}_{5}$ : C, 51.77; H, 2.97; N, 19.07. Found: C, 51.91; H, 2.90; N, 18.98.

4,5-Dimethyl-8-(4-nitrophenyl)-7-phenylpyrimido[4,5- $d]$ pyridazin-2(1H,3H,7H)-one

(7d). Orange solid, IR (KBr): 3462, 1686, 1593, 1512, $1330 \mathrm{~cm}^{-1} ;{ }^{1} \mathrm{H}$ NMR (DMSO- $\left.d_{6}, 400 \mathrm{MHz}\right): \delta=$ $10.92(\mathrm{~d}, 1 \mathrm{H}, J=1.6 \mathrm{~Hz}), 10.63(\mathrm{~d}, 1 \mathrm{H}, J=1.6 \mathrm{~Hz}), 8.17$ (d, 2H, $J=8.8 \mathrm{~Hz}), 7.57-7.50(\mathrm{~m} .6 \mathrm{H})$, 7.46-7.42 (m, 1H), 2.10, 2.09 (two s, 6H); ${ }^{13} \mathrm{C}$ NMR (DMSO-d, $100 \mathrm{MHz}$ ): $\delta=154.04,147.44$, $14.50,139.10,138.61,136.78,129.20,127.57,124.59,127.19,117.31,115.22,109.50,121.22$, 11.46; Anal. Calcd. for $\mathrm{C}_{20} \mathrm{H}_{17} \mathrm{~N}_{5} \mathrm{O}_{3}$ : C, 63.99; H, 4.56; N, 18.66. Found: C, 64.10; H, 4.45; N, 18.61 .

8-(4-Bromophenyl)-4,5-dimethyl-7-phenylpyrimido[4,5- $d]$ pyridazin-2(1H,3H,7H)-one (7e). Pale orange solid, IR (KBr): $3415,1689,1504,1382 \mathrm{~cm}^{-1} ;{ }^{1} \mathrm{H}$ NMR (DMSO- $d_{6}, 400 \mathrm{MHz}$ ): $\delta=$ $10.64(\mathrm{~d}, 1 \mathrm{H}, J=2 \mathrm{~Hz}), 10.18(\mathrm{~s}, 1 \mathrm{H}), 7.84-7.70(\mathrm{~m}, 5 \mathrm{H}), 7.47-7.44(\mathrm{~m}, 3 \mathrm{H}), 7.26-7.24(\mathrm{~m}, 1 \mathrm{H})$, $1.97(\mathrm{~s}, 6 \mathrm{H}) ;{ }^{13} \mathrm{C}$ NMR (DMSO- $\left.d_{6}, 100 \mathrm{MHz}\right): \delta=193.23,154.21,133.01,131.21,128.23,127.68$, $127.44,126.46,125.53,122.76,122.10,117.98,112.03,106.89,72.23,60.20$; Anal. Calcd. for $\mathrm{C}_{20} \mathrm{H}_{17} \mathrm{BrN}_{4} \mathrm{O}: \mathrm{C}, 58.69 ; \mathrm{H}, 4.19 ; \mathrm{N}, 13.69$. Found: C, 58.89; H, 4.02; N, 13.63.

4,5-Dimethyl-8-(naphthalen-2-yl)pyrimido[4,5-d]pyridazin-2(1H,3H,7H)-one (7f). Yellow solid, IR (KBr): 3400, 330, 1690, 1505, $1384 \mathrm{~cm}^{-1} ;{ }^{1} \mathrm{H}$ NMR (DMSO- $\left.d_{6}, 400 \mathrm{MHz}\right): \delta=10.65(\mathrm{~d}$, $1 \mathrm{H}, \mathrm{J}=1.6 \mathrm{~Hz}$ ), 10.29 (s, 1H, $J=1.6 \mathrm{~Hz}), 7.53-7.48(\mathrm{~m}, 6 \mathrm{H}), 7.44-7.40(\mathrm{~m}, 1 \mathrm{H}), 2.06,2.05$ (two s, $6 \mathrm{H}) ;{ }^{13} \mathrm{C}$ NMR (DMSO- $\left.d_{6}, 100 \mathrm{MHz}\right): \delta=154.08,147.48,139.18,138.28,131.50,129.60,129.20$, 
127.41, 126.16, 124.12, 118.92, 117.73, 111.27, 109.89; Anal. Calcd. For $\mathrm{C}_{18} \mathrm{H}_{16} \mathrm{~N}_{4} \mathrm{O}$. Found: C, 71.04; H, 5.30; N, 18.41. Found: C, 71.25; H, 5.15; N, 18.33 .

\section{Acknowledgements}

The authors gratefully acknowledge partial support of this work by the Yasouj University, Iran.

\section{References}

1. Yavari, I.; Bayat, M. J.; Sirouspour, M.; Souri S. Tetrahedron 2010, 66, 7995-7999. http://dx.doi.org/10.1016/j.tet.2010.08.016

2. Shengule, S. R.; Karuso, P. Australian J. Chem. 2011, 64, 1617-1620. http://dx.doi.org/10.1071/CH11358

3. Hulme, C.; Gore, V. Curr. Med. Chem. 2003, 10, 51-80. http://dx.doi.org/10.2174/0929867033368600 PMid:12570721

4. Turbiak, A. J.; Kampf, J. W.; Showalter, H. D. H. Tetrahedron Lett. 2010, 51, 1326-1328. http://dx.doi.org/10.1016/j.tetlet.2010.01.007 PMid:21103016 PMCid:PMC2987637

5. McTavish, D.; Young, R. A.; Clissold, S. P. Drugs 1990, 40, 543-560. http://dx.doi.org/10.2165/00003495-199040040-00005 PMid:2083513

6. Biziere, K.; Worms, P.; Kan, J. P.; Mandel, P.; Garattini, S.; Roncucci, R. Drugs Exp. Clin. Res. 1985, 11, 831-840.

PMid:3836113

7. Iizawa, Y.; Okonogi, K.; Hayashi, R.; Iwahi, T.; Yamazaki, T.; Imada, A.; Antimicrob Agents Chemother. 1993, 37, 100-105.

http://dx.doi.org/10.1128/AAC.37.1.100 PMid:8431004 PMCid:PMC187612

8. Aleeva, G. N.; Molodavkin, G. M.; Voronina, T. A. Bull. Exp. Biol. Med. 2009, 148, 54-56. http://dx.doi.org/10.1007/s10517-009-0638-4

9. Rezaei, Z.; Sharbaf, F. R.; Pourmojieb, M.; Youefzadeh-Fard, Y.; Motevalian, M.; Khazaeipour, Z.; Esmaeili, S. Acta Med. Iran 2011, 49, 701-706. PMid:22131238

10. Singh, K.; Arora, D.; Singh, K.; Singh, S. Mini Rev. Med. Chem. 2009, 9, 95-106. http://dx.doi.org/10.2174/138955709787001686

11. Grover, G. J.; Dzwomczyk, S.; Mcmullen, D. M.; Normadinam, C. S.; Sleph, P. G.; Moreland, S. J. Cardiovasc. Pharmacol. 1995, 26, 289-294. http://dx.doi.org/10.1097/00005344-199508000-00015

12. Schnell, B.; Strauss, U. T.; Verdino, P.; Faber, K.; Kappe, C. O. Tetrahedron: Asymmetry 2000, $11,1449-1453$. http://dx.doi.org/10.1016/S0957-4166(00)00081-1 
13. Aron, Z. D.; Overman, L. E. Chem. Commun. 2004, 253-265. http://dx.doi.org/10.1039/b309910e PMid:14740029

14. Byk, G.; Gettlieb, H. E.; Herscovici, J.; Mirkin, F. J. Comb. Chem. 2000, 2, 732-735. http://dx.doi.org/10.1021/cc000056p

15. Haggarty, S. J.; Mayer, T. U.; Miyamoto, D. T.; Fathi, R.; King, R. W.; Mitchison, T. J.; Schreiber, S. L. Chem. Biol. 2000, 7, 275-286. http://dx.doi.org/10.1016/S1074-5521(00)00101-0

16. Karami, B.; Khodabakhshi, S.; Eskandari, K. Tetrahedron. Lett. 2012, 53, 1445-1446. http://dx.doi.org/10.1016/j.tetlet.2012.01.024

17. Karami, B.; Khodabakhshi, S.; Nikrooz, M. Polycyclic Aromat. Compd. 2011, 31, 97-109. http://dx.doi.org/10.1080/10406638.2011.572577

18. Karami, B.; Eskandari, K.; Khodabakhshi, S. Arkicoc 2012, (ix), 76-84.

19. Karami, B.; J.Hoseini, S.; Eskandari, K.; Ghasemi, A.; Nasrabadi, H. Catal. Sci. Technol. 2012, 2, 331-338.

20. Karami, B.; Ghashghaee, V.; Khodabakhshi, S. Catal. Commun. 2012, 20, 71-75. http://dx.doi.org/10.1016/j.catcom.2012.01.012

21. Karami, B.; Farahi, M.; Khodabakhshi, S. Helv. Chim. Acta 2012, 95, 455-460. http://dx.doi.org/10.1002/hlca.201100342

22. Riley H. A.; Gray. A. R. Org. Synth. Coll. 1935, 15, 67.

23. Karami, B.; Taei, M.; Khodabakhshi, S.; Jamshidi, M. J. Sulf. Chem. 2012, 33, 65-74. http://dx.doi.org/10.1080/17415993.2011.629659 